PROCEEDINGS OF THE

AMERICAN MATHEMATICAL SOCIETY

Volume 29, Number 3, August 1971

\title{
ON THE FOURIER-ALGEBRA OF A LOCALLY COMPACT AMENABLE GROUP
}

\author{
VOLKER FLORY
}

Abstract. Let $G$ be a locally compact amenable group. The elements of the Fourier-algebra $A(G)$ are characterized with the aid of the theorem of Schoenberg-Eberlein-Eymard.

In this paper an extension of the theorem of Schoenberg-EberleinEymard is shown, which yields a characterization of the Fourieralgebra $A(G)$ of a locally compact amenable group. For abelian groups our result is due to Raouf Doss [2].

Let $G$ be a locally compact group with left-invariant Haar measure $d x$ and let $L^{p}(G), p \geqq 1$, be the Banach space of $p$-integrable complexvalued functions on $G$. By $M^{1}(G)$ we denote the convolution-algebra of the bounded Radon-measures on $G$. Let $\rho$ be the left-regular representation and $\Sigma$ the set of all (classes of) strongly continuous unitary representations of $G$.

It is known that every $\pi \in \Sigma$ in the Hilbert space $\mathfrak{S}_{\pi}$ can be lifted to a nondegenerate representation of $L^{1}(G)$ by

$$
\pi(f)=\int_{G} f(x) \pi(x) d x .
$$

If ||$\pi(f)|| \mid$ denotes the norm of the operator $\pi(f)$ on the Hilbert space $\mathfrak{S}_{\pi}$ then for every nonempty subset $\Omega \subset \Sigma\|f\|_{\Omega}=\sup _{\pi \in \Omega}\|\pi(f)\| \|$ de-

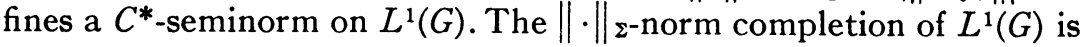
called the $C^{*}$-group algebra $C^{*}(G)$ and the completion of $L^{1}(G)$ under $\|\cdot\|_{\rho}$-norm is called the reduced $C^{*}$-group algebra $C_{\rho}^{*}(G)$.

It is known that $G$ is amenable iff $\|f\|_{\Sigma}=\|f\|_{\rho}$ for all $f \in L^{1}(G)$. In this case $C_{\rho}^{*}(G)$ is isomorphic to $C^{*}(G)$ [1]. Let $u$ be a continuous linear functional on $C^{*}(G)$. Since $\|f\|_{1} \geqq\|f\|_{\Sigma}$ for all $f \in L^{1}(G), u$ defines also a continuous linear functional on $L^{1}(G)$. Therefore the dual space $B(G)=C^{*}(G)^{\prime}$ can be considered as a subspace of $L^{\infty}(G)$. In [1] Eymard showed that $B(G)$ consists of bounded continuous functions and that $B(G)$ is a commutative Banach algebra with identity under pointwise multiplication.

\footnotetext{
Received by the editors September 30, 1970 and, in revised form, January 10, 1971.

AMS 1969 subject classifications. Primary 2265; Secondary 4680.

Key words and phrases. Theorem of Schoenberg-Eberlein-Eymard, Fourieralgebra, amenable group, convolution, representation, $C^{*}$-group algebra.
} 
By $K(G)$ denote the space of all continuous functions $f$ on $G$ with compact support $\operatorname{supp}(f)$. Then the space

$$
K * K=\left\{\sum_{i=1}^{n} f_{i} * g_{i} \mid f_{i}, g_{i} \in K(G), n \in N\right\}
$$

is contained in $B_{\rho}(G)$ and its closure $A(G)$, "the Fourier-algebra of $G$ " is a closed ideal in $B(G)$.

REMARK. If $G$ is abelian and $\hat{G}$ the character group of $G$, then $B(G)$ is the algebra of all Fourier-Stieltjes-transforms $\hat{\mu}$ of bounded Radonmeasures $\mu \in M^{1}(\hat{G})$ with $\|\hat{\mu}\|=\|\mu\|_{1}$ and $A(G)$ is exactly the algebra of all Fourier-transforms $\hat{f}$ with $f \in L^{1}(\hat{G})$ and $\|\hat{f}\|=\|f\|_{1}$.

Let $V N(G)$ be the Von Neumann-algebra of operators on $L^{2}(G)$ generated by $\rho(K(G))$. It is known that $V N(G)$ contains all operators $\rho(\mu), \mu \in M^{1}(G)$, and in [1] Eymard showed that $V N(G)$ is isometrically isomorphic to the dual space $A^{\prime}(G)$ of $A(G)$ by virtue of the isomorphism $\Phi$,

$$
\Phi: V N(G) \rightarrow A^{\prime}(G)
$$

defined by $\Phi(T)=\varphi_{T}$ with

$$
\varphi_{T}\left((f * g)^{2}\right)=(T f \mid g) \text { for } f, g \in K(G) \text { and } \check{f}(x)=f\left(x^{-1}\right) \text {. }
$$

At this the ultraweak topology of $V N(G)$ corresponds to the weak topology of the duality $\sigma\left(A^{\prime}, A\right)$.

$V N(G)$ is a $B(G)$-module, where the action of $B(G)$ on $V N(G)$ is defined as follows:

If $u \in B(G)$ and $T \in V N(G)$, then $u T$ is the uniquely determined operator $V N(G)$, for which

$$
\varphi_{u T}(v)=\varphi_{T}(u v) \quad \text { for all } v \in A(G) .
$$

We have $\|u T\|_{\rho} \leqq\|u\|\|T\|_{\rho}$. Particularly, if $T=\rho(\mu)$ for a certain $\mu \in M(G)$, then $u \rho(\mu)=\rho(u \mu)$, where $u \mu$ is the usual product of the measure $\mu$ with the function $u$.

In $[1,(2.3)(2.24)]$ Eymard generalized the theorem of SchoenbergEberlein from abelian groups to arbitrary locally compact groups:

Let $V N_{D}(G)$ be the set of all finite linear combinations $\delta=\rho\left(\sum c_{i} \delta_{x_{i}}\right)$ of Dirac measures in $V N(G)$.

A complex valued function $u$ defined on $G$ is contained in $B(G)$ iff it is continuous and if there exists a constant $M \geqq 0$ such that, for all $\delta \in V N_{D}(G)$,

$$
\begin{gathered}
\left|\sum c_{i} u\left(x_{i}\right)\right| \leqq M\left\|\rho\left(\sum c_{i} \delta_{x_{i}}\right)\right\|_{\rho}, \\
\|u\|=\sup \left\{\left|\sum c_{i} u\left(x_{i}\right)\right| ;\left\|\rho\left(\sum c_{i} \delta_{x_{i}}\right)\right\|_{\rho} \leqq 1\right\} .
\end{gathered}
$$


With the aid of this theorem it is possible to determine when an element of $B(G)$ is actually in $A(G)$ in case $G$ is amenable.

Definition. Let $B_{c}(G)$ be the set of all functions $u \in B(G)$ such that for any $\epsilon>0$ there exists a compact $K=K(\epsilon, u) \subset G$ such that $\left\|\rho\left(\sum c_{i} \delta_{x_{i}}\right)\right\|_{\rho} \leqq 1$ and $x_{i} \notin K$ implies $\left|\sum c_{i} u\left(x_{i}\right)\right|<\epsilon$.

Theorem. If $G$ is amenable, then $A(G)=B_{c}(G)$.

Proof. For $\delta=\rho\left(\sum c_{i} \delta_{x_{i}}\right) \in V N_{D}(G)$ and $u \in A(G)$ we write $\sum c_{i} u\left(x_{i}\right)=\langle u \mid \delta\rangle$.

(1) Let $u \in A(G)$ and $\epsilon>0$ be given. Since $A(G) \cap K(G)$ is dense in $A(G)$, there exists an $u_{K} \in A(G) \cap K(G)$ with compact support $K$ $=\operatorname{supp} u_{K}$ and $\left\|u-u_{K}\right\|<\epsilon$. Now let $\delta=\rho\left(\sum c_{i} \delta_{x_{i}}\right),\|\delta\|_{\rho} \leqq 1$ where $x_{i} \notin K$. Then $|\langle u \mid \delta\rangle|=\left|\left\langle u-u_{K} \mid \delta\right\rangle\right| \leqq\left\|u-u_{K}\right\|\|\delta\|_{\rho} \leqq \epsilon$. Therefore $u \in B_{c}(G)$.

(2) For a measurable set $V$ in $G$, let $\chi_{V}$ denote the characteristic function and $|V|$ the left Haar measure of $V$.

Let $u \in B_{c}(G)$ and $1 \geqq \epsilon>0$. By assumption there exists a compact $K \subset G$ such that for all $\delta \in V N_{D}(G)$ with supp $\delta \cap K=\varnothing$, one has $|\langle u \mid \delta\rangle| \leqq \epsilon\|\delta\| \rho$. Since $G$ is amenable, there exists a compact $V \subset G$ with positive Haar measure such that (cf. [4]) $|K V| /|V| \leqq 1+\epsilon$.

Now consider the function

$$
v=|V|^{-1} \chi_{K V} * \tilde{\chi}_{V} \quad \text { where } \tilde{\chi}_{V}(x)=\overline{\chi_{V}\left(x^{-1}\right)} .
$$

Then $v \in A(G)$ and $\|v\| \leqq|V|^{-1}\left\|\chi_{K V}\right\|_{2}\left\|\chi_{V}\right\|_{2} \leqq 1+\epsilon$ and for all $x \in K$ one has $v(x)=1$ and $\operatorname{supp}(v) \subset K V V^{-1}$. For $K_{1}=K V V^{-1}$ the same construction yields another function $w \in A(G)$ with the properties:

$$
\|w\| \leqq 1+\epsilon \text { and } w(x)=1 \text { for all } x \in K_{1} .
$$

Now we claim $\|u-w u\| \leqq 9 \epsilon$. To see this let $\delta \in V N_{D}(G)$ with $\|\delta\|_{\rho} \leqq 1$. Then $\|v \delta\|_{\rho} \leqq 1+\epsilon$ and $\|(1-v) \delta\|_{\rho} \leqq 2+\epsilon$ and

$$
|\langle u-w u \mid \delta\rangle| \leqq|\langle u-w u \mid v \delta\rangle|+|\langle u-w u \mid(1-v) \delta\rangle| .
$$

The first summand on the right side vanishes, because $\langle u-w u \mid v \delta\rangle$ $=\langle(v-v w) u \mid \delta\rangle$ and $v=v w$ by choice of $w$. The second summand is dominated by

$$
\begin{gathered}
|\langle u \mid(1-v) \delta\rangle|+|\langle u \mid w(1-v) \delta\rangle| \\
\leqq \epsilon(2+\epsilon)+\epsilon(1+\epsilon)(2+\epsilon) \leqq 9 \epsilon
\end{gathered}
$$

since $\operatorname{supp}((1-v) \delta) \cap K=\operatorname{supp}(w(1-v) \delta) \cap K=\varnothing$. Hence we have $\|u-w u\|<9 \epsilon$. Since $A(G)$ is a closed ideal in $B(G)$ it follows that $u \in A(G)$ and the proof is complete. 


\section{REFERENCES}

1. P. Eymard, L'algebre de Fourier d'un groupe localement compact, Bull. Soc. Math. France 92 (1964), 181-236. MR 37 \#208.

2. R. Doss, On the transform of a singular or an absolutely continuous measure, Proc. Amer. Math. Soc. 19 (1968), 361-363. MR 36 \#5619.

3. F. P. Greenleaf, Invariant means on topological groups and their applications, Van Nostrand Math. Studies, no. 16, Van Nostrand, Princeton, N.J., 1969. MR 40 \#4776.

4. H. Leptin, On locally compact groups with invariant means, Proc. Amer. Math. Soc. 19 (1968), 489-494. MR 39 \#361.

University of HeIDElberg, Heidelberg, West Germany 